\title{
Kinematics for Combined Quasi-Static Force and Motion Control in Multi-Limbed Robots
}

\author{
Krishna Shankar, Joel W. Burdick \\ California Institute of Technology, Pasadena, CA 91125 \\ \{krishna, jwb\}erobotics.caltech.edu
}

\begin{abstract}
This paper considers how a multi-limbed robot can carry out manipulation tasks involving simultaneous and compatible end-effector velocity and force goals, while also maintaining quasi-static stance stability. The formulation marries a local optimization process with an assumption of a compliant model of the environment. For purposes of illustration, we first develop the formulation for a single fixed based manipulator arm. Some of the basic kinematic variables we previously introduced for multi-limbed robot mechanism analysis in [1] are extended to accomodate this new formulation. Using these extensions, we provide a novel definition for static equilibrium of multi-limbed robot with actuator limits, and provide general conditions that guarantee the ability to apply arbitrary end-effector forces. Using these extended definitions, we present the local optimization problem and its solution for combined manipulation and stance. We also develop, using the theory of strong alternatives, a new definition and a computable test for quasi-static stance feasibility in the presence of manipulation forces. Simulations illustrate the concepts and method.
\end{abstract}

\section{INTRODUCTION}

This paper considers how a multi-limbed robot can carry out manipulation tasks involving simultaneous control of compatible position and force goals while maintaining a stable quasi-static stance in the presence of gravity. A particular motivation for this work comes from our experience with using the RoboSimian robot (see Fig. 1) to compete in the DARPA Robotics Challenge (DRC). Each of RoboSimian's four limbs incorporate 7 actuated revolute joints and three retractable fingers on the distal end of the limb. Each limb can either be used as a leg to enable locomotion, or as a limb to carry out manipulation tasks, aided by the distal fingers.

Some of the DRC tasks, such as turning a water valve handle mounted on a wall (see Fig. 1), require the vehicle to simultaneously execute a complex manipulation task involving both position and force control of the end-effector on one limb, while also maintaining stance stability throughout the task execution using the other limbs. In the valve turning example, the manipulating limb must generate a significant torque about the valve's rotational axis, while also controlling the limb and gripper to compatibly track the motion of the grasped valve handle as it rotates. We may need to coordinate all of the actuators of the entire body, not just of the manipulating limb, to generate the needed forces. Additionally the end-effector forces generated during the task must not destablize the robot's stance.
This paper introduces a new method to implement complex tasks of this sort, involving both end-effector task and motion goals, as well as stance stability. Moreover, we construct the control algorithm through a novel formulation whose locally optimal solution can be reduced to a very efficient convex program. This approach allows us to implement real-time solutions on robots having the complexity of RoboSimian. The approach's underlying formalism also allows us to develop new definitions and new criteria for the feasiblility of quasi-static stances under the influence of manipulation forces.

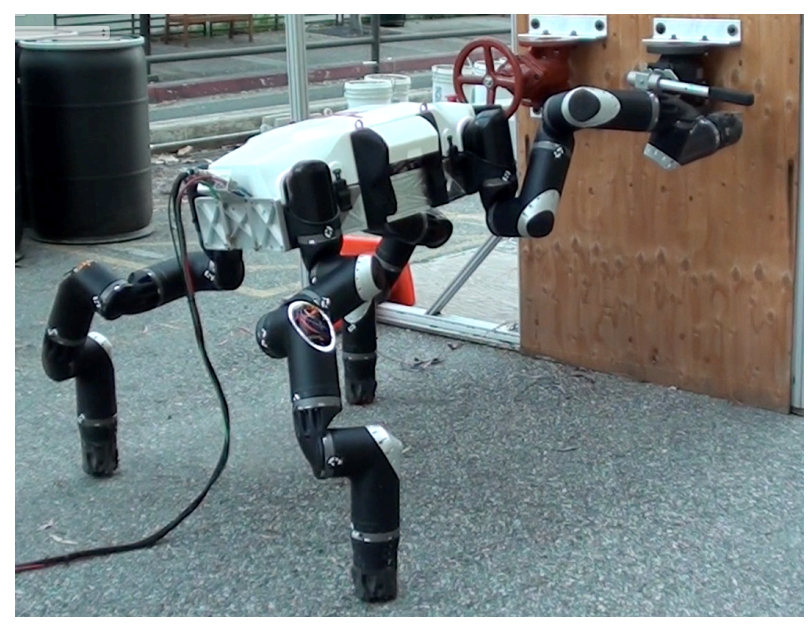

Fig. 1. Photograph of the Four-Limbed RoboSimian Robot used by the JPL/Caltech team in the DARPA Robotics Challenge. This snapshot shows RoboSimian turning a value on the vertical wall in the background using one of its limbs, while maintaining quasi-static stance stability using its other three limbs.

Relation to Prior Work. Our problem is closely related to several important and historical problems in the field of robotics. There is a vast prior literature on simultaneous control of end-effector force and position during manipulation tasks using a fixed-based robot manipulator [2]. Numerous frameworks for this problem have been introduced, such as hybrid position-force control [3], impedance control [4], stiffness control [5], [6], and operational space control [7]. Each of these approaches in turn has a rich literature concerning the design, analysis, and control of manipulation tasks within the respective framework. The method put forth in this paper can be seen as a form of stiffness or admittance control for multi-limbed robots that also incorporates stance stability 
criteria into the formulation. We also use a novel Quadratic Programming (QP) approach to formulate the problem. We have shown in previous work [1], [8] that such a framework can very efficiently solve the complex kinematic coordination problems that arise in multi-limbed robot coordination tasks.

Our approach can also be considered as a form of whole body manipulation [9] and mobile manipulation [10]-[12] for the particular class of quasi-statically stable legged robots. As opposed to fixed based manipulators, where stability is not an issue, or wheeled-based manipulators where stability analysis is straightforward, manipulation by multi-limbed walking robots must also incorporate stance stability into the overall manipulation planning process. We provide new explicit results on quasi-static stability for the class of problems defined above.

While this paper considers quasistically stable robots (with $n \geq 3$ legs on the ground at all times), humanoid robots must also contend with manipulation tasks while maintaining postural stability, and previous studies have analyzed and developed techniques for bipedal robots to carry out manipulation [13]-[15]. Theoretical advances in Convex Programming, and the associated introduction of efficient numerical optimization codes, allow us to propose new approaches which have not only serious computational speed advantages, but also allow added flexibility and generality in specifying the task objectives. We are not the first to propose the use of Convex Optimization or Quadratic Programming techniques for local motion planing of highly articulated mechanisms. For a review of related work, see [8]. We do not consider dynamic effects in this paper. However, many (e.g. [16], [17]) have obtained controllers for full dynamic models of humanoids with contact from simple convex QPs, for balancing and walking.

Structure of the Paper. Section II summarizes the motionforce task compatibility and stiffness-compliance modeling assumptions that underly the proposed method. Section III introduces our optimization-based framework for task control in the context of a fixed base serial chain manipulator mechanism. Section IV reviews some basic and essential kinematics of multi-limbed mechanisms that we introduced in previous work [1], while Section V extends the framework of Sections II-IV to the multi-limbed robot case, while incorporating quasi-static stance constraints. Section VI uses the theory of strong alternatives to analyze the problem of force balance in a multi-limbed robot working under both manipulation and stance constraints. An explict set of criteria is derived to describe the conditions under which a multilegged robot would not be able to maintain a quasi-static stance in the presence of manipulation forces.

\section{TASK COMPATIBILITY AND COMPliAnCE}

The section describes our procedure to encode simultaneously feasible force and velocity goals. Our formulation adopts the invariant form [18] of the classical Raibert/Craig hybrid position-force control [3] framework, and combines it with an assumed compliance model of the environment.
We assume that a set of constraint or task wrenches to be controlled by application of end-effector forces are given. These task wrenches are assumed to lie in a wrench space, $W_{\text {task }}$, having integer dimension, $N_{W}$ :

$$
W_{\text {task }}=\operatorname{span}\left[\begin{array}{lll}
W_{1} & \cdots & W_{N_{W}}
\end{array}\right] \triangleq B_{W}\left[\begin{array}{c}
\omega_{1} \\
\vdots \\
\omega_{N_{W}}
\end{array}\right] \text {, }
$$

for all $\omega_{i} \in \mathbb{R}, i=1, \ldots, N_{w}$. The vector $W_{i}$ is the $i^{t h}$ basis element for the wrenches associated with an $l$-dimensional task space $(l=3$ for planar tasks, and $l=6$ for spatial tasks). We assume that $N_{w} \leq l$. We term the matrix $B_{W}$ the wrench basis matrix for the task, as it defines the space of wrenches to be controlled.

The velocity (or infinitesimal displacement) task goals must reside in a compatible $N_{V}$-dimensional space of velocities [19]:

$$
V_{\text {task }}=\operatorname{span}\left[\begin{array}{lll}
\xi_{1} & \cdots & \xi_{N_{V}}
\end{array}\right]=D_{V}\left[\begin{array}{c}
v_{1} \\
\vdots \\
v_{N_{V}}
\end{array}\right]
$$

where $N_{V}+N_{W}=l$. The matrix $D_{V}$ is said to be the $t$ wist basis for the manipulation task. Each twist basis vector, $\xi_{j}$ must have the following compatibility relationship with each element of the wrench basis [18]:

$$
\xi_{j} \cdot W_{i}=0
$$

for all $i=1, \ldots, N_{W}$ and $j=1, \ldots, N_{V}$. When the twist and wrench basis elements are represented in screw coordinates, the associated screws must be reciprocal to each other. Whenever this compatibility is violated, either the goals are antagonistic (for some $i, j, \quad W_{i} \cdot V_{j}<0$ ) or infeasible (for some $i, j, W_{i} \cdot V_{j}>0$ ) [20].

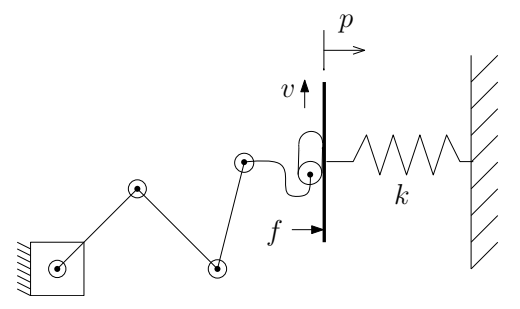

Fig. 2. A Simple Model for Compliance

In general, a wrench basis $B$ maps a contact wrench $(f)$ to a full wrenches $F$ as $F=B f$, and a twist basis $D$ maps a contact velocity $v$ to a full twist $V$ as $V=D v$.

Next, we adopt a specific relationship between forces and motions when the end-effector is in contact with its environment. We initially assume a simple compliance model that relates task wrenches at the end-effector to velocities (or infinitesimal displacements) of the end-effector along directions defined by the wrench basis.

For simplicity, let us first suppose that the robot interacts with a rigid surface that in turn is attached to a spring, as shown in Fig. 2. The robot contacts the surface at position $p$ in the world frame, and the robot applies a force $f$ along the 
normal to the rigid surface. Assuming the spring is linear, the force applied by the robot and position of the contact satisfy Hooke's law,

$$
f=k p
$$

were $p$ represents displacement from some initial contact position given by $p(0)=0$. To establish and maintain a constant task force $f_{d}$, the manipulator must move the contact to $p=\frac{1}{k} f_{d}$.

In practice, we may want to apply an arbitrary wrench (i.e., a combination of forces and torques, and not a simple Cartesian force) at the contact. In order to extend this very simple linear compliance to handle this more general case, recall that Poinsot's theorem states that every wrench can be interpreted as a 'screw' force - the combination of linear force along an axis and a torque about the same axis. We hypothesize a 'screw-spring' relationship between the movement of the end-effector contact along a screw axis to the wrench produced by this movement (as illustrated by Fig. 3). Let $\xi$ (a unit twist) describe a particular screw displacement. Then the contact wrench, $F$, as a function of end-effector position $p$ along this screw is given by

$$
F=(k p) \xi
$$

Let the intial wrench at the end-effector be given by $F_{0}$,

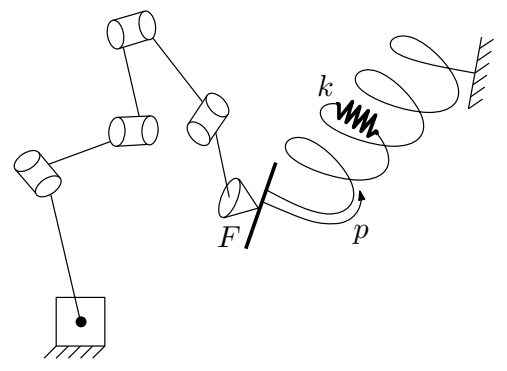

Fig. 3. Extending the Model to 3D

corresponding to position $p_{0}=0$, and suppose the desired wrench is $F_{d}$. The change in the wrench to be applied by the robot is $\Delta F=F_{0}-F_{d}$. With this assumption, $p_{d} \xi=$ $-\frac{1}{k} \Delta F$; the desired wrench can be realized by moving the contact point along $p_{d} \xi$.

More generally, suppose we can compute

$$
\Delta F=F_{\text {meas }}-F_{d}
$$

where $F_{\text {meas }}$ is the measured wrench, during execution. Then, we can achieve the desired wrench by moving the endeffector with velocity

$$
V=-\frac{\mu}{k} \Delta F
$$

where $\mu$ is an arbitrary proportional gain. For convenience, we set $\mu=1$ henceforth.

To extend this principle to the case of multiple contact wrenches, suppose that a manipulator is required to achieve a task wrench $W_{\text {task }}$ lying in the span of $B_{W}=$ $\left[W_{1} \ldots W_{N_{W}}\right]$. Since each of the $W_{i}$ are associated with a different contact, they may each be endowed with a different stiffness $k_{i}>0$. Define a stiffness matrix to have the form

$$
K=\left[\begin{array}{ccc}
k_{1} & 0 & 0 \\
0 & \ddots & 0 \\
0 & 0 & k_{N_{w}}
\end{array}\right]
$$

In general, the only requirement on a stiffness matrix $K$ is that it is positive semidefinite. However, the wrench basis can always be selected so that it is diagonal and positive as in (3).

Now, suppose that the end-effector moves along a screw $\xi$. Let

$$
p_{i}=\left(\xi^{T} W_{i}\right) p
$$

it represents the displacement along the direction of $W_{i}$. Then, the net contact wrench is

$$
F=\sum_{i=1}^{N_{W}} k_{i} p_{i} W_{i}=p K B_{W} B_{W}^{T} \xi
$$

Again, letting $\Delta F=F_{\text {meas }}-W_{\text {task, }}$, we can achieve the desired wrench by moving with a velocity $V$ satisfying

$$
B_{W} K B_{W}^{T} V=-\Delta F
$$

Eq. (4) is a general linear compliance relationship that allows us to understand how motions result in forces; going forward, we use this relationship in planning motion locally, to achieve position and force goals. The relationship (4) is well known; for example, it is along the same lines as the compliance models in [21] and [6].

We recognize that there are shortcomings with our assumed model for the environment.

1) The model assumes a linear elastic response of the environment. However, non-linear stiffness or compliance models are readily incorporated via linearization of the elastic model [21], [22] to construct a configuration dependent stiffness matrix.

2) In most situations, the stiffness of the contact is not a priori known accurately. In fact, very poor assumptions about the environment's compliance can lead to catastrophic results. To ensure safety, the assumed contact stiffness should be assumed larger than the true contact stiffness. The price to pay for this conservative modeling approach is the potentially increased time needed to reach a desired contact wrench. E.g., if the true stiffness is $k$ and we assume it to be $\tilde{k}$, if we do not measure force, then we apply a force equal to $\frac{k}{\tilde{k}} f$. If an accurate measurement, $f_{c u r}$, of the force is available, the desired force can be regulated with a simple motion control law (e.g. law $\dot{p}=\frac{1}{\tilde{k}}\left(f_{d}-f_{\text {cur }}\right)$ results in exponential asymptotic convergence to the desired force.)

Our approach is motivated by the following practical considerations in working with the RoboSimian vehicle, and many similar robots. To realize a very high strength to weight ratio, the motors in RoboSimian's legs are highly geared (using harmonic drives), preventing us from exercising accurate control of joint torques. However, very fine control over joint 
displacements are possible, suggesting that we need a control design approach which models the relationship between joint motions and end-effector forces. Moreover, the distal tips of each RoboSimian limb are covered with a thick compliant rubbery pad, which can be accurately modeled using the simple methods of this section. In the language of impedance control, we must design an admittance for such systems.

\section{Simultaneous Force and Position Control: FIXED BASE MANIPULATOR}

To gain intuition for the problem we wish to solve with a multi-limbed robot, we first consider the simpler case of a fixed base manipulator, and the corresponding simplified problem. For a fixed base serial chain manipulator with the ability to measure end-effector wrenches, we wish to implement a local planning and control system which solves the following word problem:

How do we move our joints so that

1) The end effector moves in the direction of a pose goal along the subspace of allowed motions

2) The manipulator controls the applied wrenchs along the allowed wrench subspace, based on a compliance model.

3) The resulting manipulator motion is minimal when there are redundant degrees of freedom.

We can formalize the solution to this intuitive word problem as the following constrained minimization:

$$
\min _{\dot{\theta}} \frac{\alpha}{2}\left\|v-v_{d}\right\|_{2}^{2}+\frac{\beta}{2}\left\|\Delta f-\left(f_{d}-f_{\text {meas }}\right)\right\|_{2}^{2}+\frac{\gamma}{2}\left\|\dot{\theta}_{2}^{2}\right\|
$$

subject to $\quad D_{V}^{T} V=v$

$$
\begin{aligned}
& B_{W}^{T} V=K^{-1} \Delta f \\
& J(\theta) \dot{\theta}=V
\end{aligned}
$$

Where $\alpha, \beta$ and $\gamma$ are positive weighting coefficients, $B_{W}$ is a wrench basis that spans the space of wrenches that are to be applied by the end-effector, and $D_{V}$ is a twist-basis whose range includes the compatible subspace of end-effector velocities. $f_{d}$ and $f_{\text {meas }}$ are the desired and measured wrenches in the basis $B_{W}$ 's coordinates. The quantity $\left(f_{d}-f_{\text {meas }}\right)$ is the end-effector wrench error, expressed in the wrench basis, which should be controlled to zero by motions of the manipulator. The coefficients $\alpha$ and $\beta$ trade off the relative importance of errors in the velocity and wrench goals, while $\gamma$ weights the importance of using a minimal motion to solve the given problem. The first two constraints ensure the compatibility relations described in the last section, and we require that $B_{W}$ and $D_{V}$, when expressed in a common frame, satisfy the relationship $B_{W}^{T} D_{V}=0$. The last constraint describes the manipulator's kinematic relationship between joint motions and end-effector velocities.

To simplify the form of the problem and its solution, define

$$
\eta \triangleq \alpha D_{V} v_{d}+\beta B_{W} K^{-1}\left(f_{d}-f_{\text {meas }}\right) .
$$

which can be interpreted as the weighted instantaneous endeffector displacement which moves towards the task goals. With this definition, the constrained minimization problem can be converted into an unconstrained minimizaton problem:

$$
\min _{\dot{\theta}} \frac{1}{2} \dot{\theta}^{T}(P+\gamma I) \dot{\theta}-\eta^{T}(J \dot{\theta})
$$

where

$$
P=J(\theta)^{T}\left(\alpha B_{W}^{T} B_{W}+\beta D_{V}^{T} D_{V}\right) J(\theta) .
$$

It can be shown that a solution to (5) exists whenever $\alpha, \beta$, $\gamma$ are nonnegative. When they are all positive, the solution at each instant is given by

$$
\dot{\theta}^{*}=(P+\gamma I)^{-1} J^{T} \eta
$$

The resulting motion $J(\theta) \dot{\theta}^{*}$ can be interpreted as a weighted or oblique projection of $\eta$ onto the range of $J(\theta)$. The extent to which the force and motion goals are locally satisfied by $\dot{\theta}^{*}$ is governed by the ratio of $\alpha$ to $\beta$. Moving according to $\dot{\theta}^{*}$ gives us a method to locally step towards achieving the task goal. In practice, this solution could be used iteratively for planning and control, or to bias samples in a randomized planner, and thereby make global search attempts tractable.

\section{Review of Multi-Limbed Robot Kinematcs}

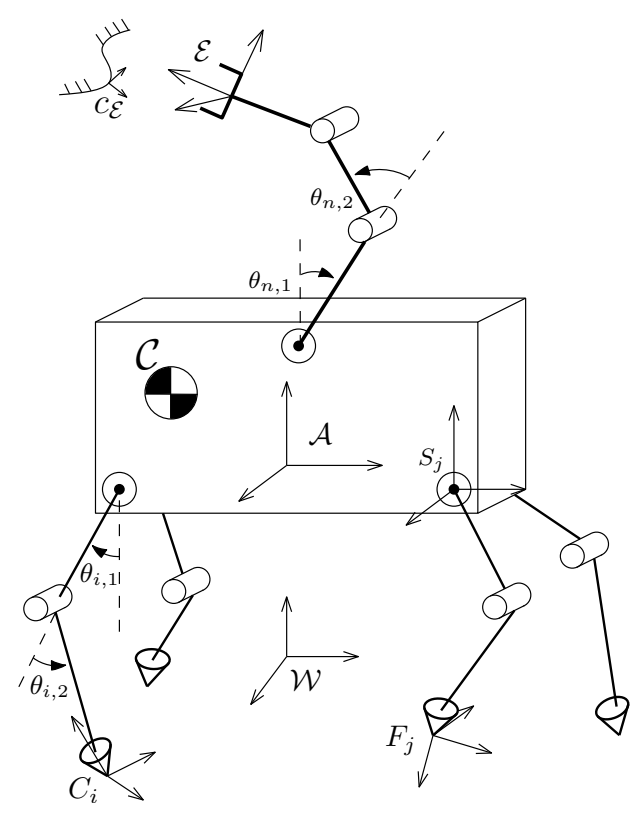

Fig. 4. Key Reference Frames for Stance and Reach.

Although multi-limbed stance and reach kinematics is the subject of our previous work [1], this section briefly reviews some key concepts that are crucial to the present work. We assume that the robot has $N \geq 4$ limbs so that it can maintain balance in a quasi-static sense as one or more limbs engage in manipulation tasks. As shown in Fig. 4, we assume a fixed world frame, $\mathcal{W}$, and an abdomen frame, $\mathcal{A}$, attached to the robot's body. The frame attached to the manipulating end-effector is $\mathcal{E}$ (extending the model and methods to accommodate multiple, simultaneously manipulating limbs is straightforward). A shoulder frame, $S_{i}$, is situated where each limb attaches to the body, and can be thought of as the 
base frame of the $i^{\text {th }}$ limb mechanism. The joint angles in the $i^{\text {th }}$ limb are denoted by $\theta_{i}=\left(\begin{array}{lll}\theta_{i, 1} & \ldots & \theta_{i, n_{i}}\end{array}\right)^{T}$, where $n_{i}$ is the number of joints in the $i^{\text {th }}$ limb. Joint angles in the manipulating limb will be denoted by $\theta_{\mathcal{E}}$. Contact frames $c_{i}, i=1, \ldots, M$ (where $M \geq 3$ ) are fixed to the environment where each of the $M$ limbs makes contact with the world. Note that if the manipulating limb makes contact, $M$ includes the manipulating limb. The $z$-axis of the $c_{i}$ frame points along the inward normal to the surface, and the $x$ and $y$-axes are tangent to the surface. The end-effector's contact frame is denoted $c_{\mathcal{E}}$, and is attached to the task surface. The origin of the center of mass is at $\mathcal{C}$.

Each contact with world has a wrench basis $B_{c_{i}}$ that spans the wrenches that the contact can sustain ${ }^{1}$. Forces at the ground contacts $f=\left[f_{1} \ldots f_{M}\right]^{T}$ are related to the net wrench at the abdomen as seen abdomen frame, $F_{\mathcal{A}}$, according to [1]

$$
F_{\mathcal{A}}=S f
$$

where $S$ is known as the stance map [1], and is given by

$$
S=-\left[\begin{array}{llll}
\operatorname{Ad}_{g_{\mathcal{A} c_{1}}^{-1}}^{T} B_{c_{1}} & \cdots & \operatorname{Ad}_{g_{\mathcal{A} c_{M}}^{-1}}^{T} & B_{c_{M}}
\end{array}\right],
$$

where $\operatorname{Ad}_{g_{a b}}$ transforms a velocity expressed in frame $b$ to one expressed in frame $a$ [19]. The motion of the abdomen is a function of joint motions, and is given by

$$
J_{\mathcal{S}}\left(x_{0}, \vec{\theta}\right) \dot{\vec{\theta}}=S^{T} V_{\mathcal{W A}}^{\mathcal{A}}
$$

where $J_{\mathcal{S}}\left(x_{0}, \vec{\theta}\right)$ is the Stance Jacobian [1]:

$$
\left[\begin{array}{cccc}
B_{c_{1}}^{T} \operatorname{Ad}_{g_{s_{1} c_{1}}}^{-1} J_{1}\left(\vec{\theta}_{1}\right) & & 0 \\
& \ddots & & \\
0 & & B_{c_{M}}^{T} \operatorname{Ad}_{g_{s_{M} c_{M}}}^{-1} J_{M}\left(\vec{\theta}_{M}\right)
\end{array}\right] \text {. }
$$

The variable $x_{0}$ includes contact locations, orientations and other information about the robot's current posture. The velocity $V_{a b}^{c}$ should be read as 'the velocity of $b$ with respect to $a$ as seen in frame $c$.

In the equations above, and throughout the paper, $\dot{\theta}$ is the vector of all of the joint rates in the supporting legs, while $\dot{\Theta}$ is the vector of joint rates including those of the legs as well as those in the manipulating arm. With this in mind, the velocity of a manipulating end-effector is given by

$$
J_{\mathcal{R}}\left(\Theta, x_{0}\right) \dot{\Theta}=S^{T} V_{\mathcal{W E}}^{\mathcal{A}}
$$

where

$$
J_{\mathcal{R}}\left(\Theta, x_{0}\right)=\left[J_{\mathcal{S}}\left(x_{0}, \vec{\Theta}\right) S^{T} J_{\mathcal{E}}\left(\vec{\theta}_{\mathcal{E}}\right)\right]
$$

is the reach Jacobian [1], which relates the motion of the joints in both the manipulating limb and the supporting limbs (which are restricted so as to maintain the feet contacts at a fixed location) to the motion of the end-effector.

\footnotetext{
${ }^{1}$ For more information on wrench bases of frictional contact, see [19].
}

\section{Simultaneous Force AND Position CONTROL FOR LEGGED ROBOTS}

We now extend the ideas of Section III and IV to the case of multi-limbed robots which use one or more limbs to carry out simultaneous velocity and force manipulation tasks, while also maintaining a static equilibrium. We restrict our analysis to the case of one manipulating limb, with the remaining limbs providing support. The manipulating limb contacts the world, with the goal of imparting wrenches to its contact as defined by the wrench basis $B_{W}$. Now we can express the manipulation task wrenches in the abdomen frame as

$$
F_{\mathcal{E}}^{\mathcal{A}}=\operatorname{Ad}_{g_{\mathcal{E} \mathcal{A}}}^{T} B_{W} f_{\mathcal{E}}
$$

where $f_{\mathcal{E}}$ is the manipulating contact force expressed in the coordinates of $B_{W}$. We also define a twist basis $D_{V}$ that compliments $B_{W}$ and satisfies the local compatibility condition. In [1], we showed that an end-effector motion can arise from some combination of abdomen movement and free-limb movement. However, for free-limb forces, wrenches applied by the end-effector must be resisted by the supporting limbs in order to maintain static equilibrium.

To accomodate a manipulating limb in contact, it must be accounted for in the stance map,

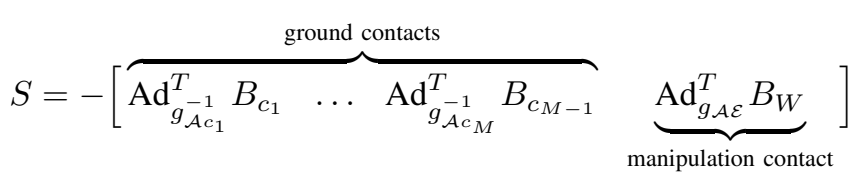

as well as in the vector of contact forces, $f=$ $\left[\begin{array}{llll}f_{1} & \ldots & f_{M-1} & f_{\mathcal{E}}\end{array}\right]^{T}$. The Stance Jacobian too must include the block diagonal term associated with the manipulating limb (the index $M$ in (6) is replaced by $\mathcal{E}$ ).

Definition 1: A multi-legged robot is in static equilibrium if there exists $f$ satisfying

$$
\begin{aligned}
& S f=\operatorname{Ad}_{g_{\mathcal{C A}}} \mathcal{G} \\
& f \in \mathrm{FC}
\end{aligned}
$$

Where $\mathrm{f}$ is a vector of contact frame forces including those of the manipulating limb, $\mathcal{G}$ is the gravity wrench acting on the origin of the center of mass frame $\mathcal{C}$ and $\mathrm{FC}$ is the friction cone ${ }^{2}$.

We can use this definition to obtain the set $\mathcal{F}_{\mathcal{E}}$ of end-effector forces that can possibly be produced by the mechanism in its current configuration

$$
\mathcal{F}_{\mathcal{E}}=\{x-\mathcal{G} \mid x \in \mathcal{R}(S)\} .
$$

where $\mathcal{R}(A)$ is the range of matrix $A$. In general however, the set of feasible manipulating forces $F_{\mathcal{E}}$ depends not only on the wrench basis $B_{W}$ but also on the nature of contact of the supporting legs, defined by the friction cone. The following provides some general conditions under which a stance can produce any manipulating force:

\footnotetext{
${ }^{2}$ The friction cone is the set of forces that satisfy the chosen contactfriction model For backround and examples, see [19]
} 
Proposition 1: A legged robot can sustain any endeffector manipulating force, $f_{\mathcal{E}}$, if and only if $S$ is surjective, and there exists $f_{G}$ satisfying $S f_{g}=\mathcal{G}$, and there exists an internal force $f_{\text {int }} \in \mathcal{N}(S)^{3}$ such that $f_{\text {int }} \in \operatorname{int}(F C)$.

Proof $^{4}$. $\Leftarrow$ Let $f_{\mathcal{E}} \in \mathrm{FC}_{\mathcal{E}}$. Since $S$ is surjective, there exists $f^{\prime} \in \mathrm{FC}$ such that $S f^{\prime}+B_{W} f_{\mathcal{E}}=0$. Now, since $f_{\text {int }} \in$ $\mathrm{FC}$ and since $\mathrm{FC}$ is a cone, for any $a \geq 0, a f_{\text {int }} \in \mathrm{FC}$. For $a$ large enough, $a f_{n}+f^{\prime} \in \mathrm{FC}$ since the friction cone is closed and since $f_{n} \in \operatorname{int}(F C)$. Then, we have $S\left(f_{g}+\right.$ $\left.\left[\begin{array}{ll}f^{\prime}+a f_{n} & f_{\mathcal{E}}\end{array}\right]^{T}\right)=G$.

$\Rightarrow$ Suppose that we have $S f+B_{W} f_{\mathcal{E}}=G$. Pick $f_{1} \in$ $\operatorname{int}(\mathrm{FC})$ so that $S f_{1}=F \neq 0$ and select $f_{2}$ satisfying $S f_{2}=$ $-F$. Then, set $f_{\text {int }}=f_{1}+f_{2}$. We now have $S f_{\text {int }}=0$ and $f_{\text {int }} \in \operatorname{int}(\mathrm{FC})$.

Now that we know how forces at the contacts result in forces at the abdomen, we can ask how do motions of the abdomen result in forces on the abdomen? It makes little sense to define a stiffness that relates abdomen motions to wrenches on the abdomen directly as we did for the end effector. Instead, we must obtain a compliance relationship between contact forces at the feet or end-effectors and abdomen motions.

Let the stiffness matrix for the $i^{\text {th }}$ limb stance limb that makes contact be $K^{i}$ (see equation (3) in section II), defined with respect to its contact frame. Contact frame velocities $\nu_{i}$ and contact frame forces $f_{i}$ are related by

$$
K^{i} \nu_{i}=f_{i}
$$

More generally, if the manipulating limb makes contact, then we must include the effect of the manipulating limb's motion in the subspace defined by the basis $B_{W}$. Contact velocities and forces (in the coordinates of $B_{W}$ ) are related by

$$
f_{\mathcal{E}}=K^{\mathcal{E}} \nu_{\mathcal{E}}
$$

In [1], we showed that instantaneous contact velocites are related to abdomen velocities by

$$
S^{T} V_{\mathcal{W} \mathcal{A}}^{\mathcal{A}}=\nu
$$

where $\nu=\left[\begin{array}{llll}\nu_{1} & \ldots & \nu_{M-1} & \nu_{\mathcal{E}}\end{array}\right]^{T}$. Therefore, we get the following compliance relationship between abdomen wrenches and velocities:

$$
K_{\mathcal{S}} V_{\mathcal{W} \mathcal{A}}^{\mathcal{A}}=\Delta F_{\mathcal{A}}
$$

Where we call $K_{\mathcal{S}}$ the stance stiffness matrix:

$$
K_{\mathcal{S}}=S \tilde{K} S^{T}
$$

where

$$
\tilde{K}=\left[\begin{array}{cccc}
K^{1} & 0 & 0 & 0 \\
0 & \ddots & 0 & 0 \\
0 & 0 & K^{M} & 0 \\
0 & 0 & 0 & K^{\mathcal{E}}
\end{array}\right]
$$

\footnotetext{
${ }^{3} \mathcal{N}(A)=$ Null Space of matrix A.

${ }^{4}$ This proposition is similar in nature to Proposition 5.2 in [19] and the proof is along the same lines
}

When the manipulating limb is not in contact, $\tilde{K}$ will not include the last diagonal block representing the manipulating contact stiffness. Note that in general, $K_{\mathcal{S}}$ is invertible, since the stiffness matrices $K_{i}$ and $K_{\mathcal{E}}$ are positive definite, by convention (see section II), and since $S$ will generally be onto ${ }^{5}$.

Now we address the issue of achieving a manipulation goal that requires moving from one pose to another, while achieving and maintaining a wrench. Compared to the fixed base manipulator of Section III, we must account for gravity and for the motion of the supporting legs. We must also include the compliance model from Section II to relate forces and velocites.

In words we want to solve the following problem: given a combined pose and wrench goal for the manipulating limb, move the manipulating and supporting limbs' joints so as to

- Ensure that the end-effector moves towards the pose goal, within the motion subspace defined by $D_{V}$.

- Control the end-effector wrench to lie with the wrench subspace defined by $B_{W}$, towards achieving a desired wrench.

- Ensure that static stance equilibrium is achieved by regulating the net wrench at the abdomen due to gravity.

- move minimally when the robot is redundant.

$$
\begin{array}{cl}
\min _{\dot{\Theta}} & \alpha\left\|v_{\mathcal{E}}-v_{\mathcal{E}, d}\right\|_{2}^{2}+\beta\left\|\Delta F_{\mathcal{A}}-F_{\text {meas }}\right\|_{2}^{2} \\
& +\left\|\Delta f_{\mathcal{E}}-\left(f_{\mathcal{E}, d}-f_{\mathcal{E}, \text { meas }}\right)\right\|_{2}^{2}+\gamma\|\dot{\Theta}\|_{2}^{2} \\
\text { subject to } \quad & S^{T} V_{\mathcal{W} \mathcal{E}}^{\mathcal{A}}=J_{\mathcal{R}} \dot{\Theta} \\
& D_{V}^{T} \operatorname{Ad}_{g_{\mathcal{E} \mathcal{A}}} V_{\mathcal{W} \mathcal{E}}^{\mathcal{A}}=v_{\mathcal{E}} \\
& B_{W}^{T} \operatorname{Ad}_{g_{\mathcal{E} \mathcal{A}}} V_{\mathcal{W} \mathcal{E}}^{\mathcal{A}}=K_{\mathcal{E}}^{-1} \Delta f_{\mathcal{E}} \\
& S^{T} V_{\mathcal{W} \mathcal{A}}^{\mathcal{A}}=J_{\mathcal{S}} \dot{\theta} \\
& K_{\mathcal{S}} V_{\mathcal{W} \mathcal{A}}^{\mathcal{A}}=\Delta F_{\mathcal{A}}
\end{array}
$$

where $F_{\mathcal{A}}$ is the net wrench on the abdomen, $F_{\text {meas }}$ is the measured wrench at the abdomen,

$$
F_{\text {meas }}=S f_{\text {meas }}-\mathcal{G}
$$

with $f_{\text {meas }}$ being the vector of measured contact wrenches at the supporting feet and $\mathcal{G}$ the gravity wrench as seen in the abdomen frame. $v_{\mathcal{E}, d}$ is the motion goal for the end-effector expressed in the twist basis $D_{V}$ and $\Delta f_{\mathcal{E}}$ is the desired change in force written in the wrench basis $B_{W}$, as seen in the abdomen frame. $f_{\mathcal{E} \text {, meas }}$ and $f_{\mathcal{E}, d}$ are the measured and desired end effector forces respectively, in the task wrench basis' coordinates. The matrices $K_{\mathcal{E}}$ and $K_{\mathcal{S}}$ are the endeffector and stance stiffness matrices respectively. All of the full wrenches above and henceforth are expressed in the abdomen frame.

Conceptually, the solution to (9) provides a proportional control law that will drive error in position and force to zero, locally. To see this more clearly, we can simplify the problem

\footnotetext{
${ }^{5}$ when it is not, the robot is not force resistant [1], and is in a poor configuration for manipulation
} 
above by making the relationship between variables explicit. Let

$$
\eta_{\mathcal{E}}=\alpha D_{V} v_{\mathcal{E}, d}+\beta B_{W} K_{\mathcal{E}}^{-1}\left(f_{\mathcal{E}, d}-f_{\mathcal{E}, \text { meas }}\right),
$$

and let

$$
\eta_{\mathcal{A}}=K_{\mathcal{S}}^{-1} F_{\text {Meas }}
$$

Then, the problem (9) can be written as

$$
\begin{array}{cl}
\min _{\dot{\Theta}} & \alpha\left\|V_{\mathcal{W E}}^{\mathcal{A}}-\eta_{\mathcal{E}}\right\|_{2}^{2}+\beta\left\|V_{\mathcal{W} \mathcal{A}}^{\mathcal{A}}-\eta_{A}\right\|_{2}^{2}+\gamma\|\dot{\Theta}\|_{2}^{2} \\
\text { subject to } & S^{T} V_{\mathcal{W} \mathcal{E}}^{\mathcal{A}}=J_{\mathcal{R}} \dot{\Theta} \\
& S^{T} V_{\mathcal{W} \mathcal{A}}^{\mathcal{A}}=J_{\mathcal{S}} \dot{\theta}
\end{array}
$$

Proposition 2: The problem (10) has a solution whenever $\alpha, \beta$ and $\gamma$ are non-negative. It has a unique solution whenvever $\alpha, \beta$ and $\gamma$ are positive, there is no motion that keeps both the abdomen and center of mass stationary, and

$$
\operatorname{dim}\left(\mathcal{R}\left(D_{V}\right)\right)+\operatorname{dim}\left(\mathcal{R}\left(B_{W}\right)\right)=6 .
$$

The proof of this proposition follows readily from writing down the KKT conditions for (10), and considering the rank of the resulting 'KKT Matrix', that relates the optimal values of $\dot{\Theta}$ and its dual to the problem data (see [23]).

Note that the problems (9) and (10) do not include friction cone contstraints at the contacts. The local analysis (Prop. 2) in the presence of the friction cone constraints is the same when forces are interior to the friction cone. When the forces are on the boundary, local feasibilty depends on the particular friction model in use. We address the issue of feasibility with respect to the friction cone and actuator limits in the following section.

\section{Stance Feasibility and Alternatives}

In [1] we used a standard geometric notion of quasi-static balance: in a quasi-statically stable stance, the center of mass remains over the support region defined by the location of the legs' contacts with the supporting terrain. However, if we want to account for end-effector forces and torque limits we must take a different approach, since large end-effector forces and excess joint torques can destabilize the robot.

We start by asking what it means to be statically stable when we can measure end-effector wrenches. Fundamentally, there should be no net forces acting on the robot mechanism. This implies that all of the contact forces (at both the manipulating end-effector and at the ground contacts) must be balanced with the wrench applied on the mechanism due to gravity. Moreover, the robot must be able to balance these forces within its actuator force or torque limits. Using the kinematics from the previous section, this balance requirement can be posed as the following feasibility problem:

$$
\begin{aligned}
\text { find } & f \\
\text { subject to } & S f+\operatorname{Ad}_{g_{\mathcal{C A}} \mathcal{G}}^{T}=0 \\
& -\tau_{M} \leq J_{S}^{T} f \leq \tau_{M} \\
& f \in \mathrm{FC}
\end{aligned}
$$

where there are symmetric torque limits with magnitude $\tau_{M}$ on the joints. To better understand the meaning of this problem, and to understand exactly when or why we fall, we explore the strong alternative to this feasibilty problem - as the name suggests, this is an alternative feasibility problem that certifies the infeasibility of the current one. The alternative arises from posing the fesibility problem as a constrained minimization (with a constant objective function), and computing the conditions under which a solution exists; the negation of these conditions is an alternative. ${ }^{6}$

The alternative feasibility problem is:

$$
\begin{array}{cl}
\text { Find } & V_{\mathcal{W A}}^{\mathcal{A}}, \dot{\theta} \\
\text { subject to } & \left(V_{\mathcal{W A}}^{\mathcal{A}}\right)^{T}\left(\operatorname{Ad}_{g_{\mathcal{C} \mathcal{A}}}^{T} \mathcal{G}\right)>\dot{\theta}^{T} \tau_{M} \\
& S^{T} V_{\mathcal{W A}}^{\mathcal{A}}=J_{s} \dot{\theta}+\nu \\
& \nu \in \mathrm{FC}^{*}
\end{array}
$$

where $\mathrm{FC}^{*}$ is the dual to the friction cone given by

$$
\mathrm{FC}^{*}=\left\{\nu \mid \nu^{T} f \geq 0 \text { for all } f \in \mathrm{FC}\right\}
$$

and represents a set of contact velocities that result in slip (the instantaeous power generated by these velocities is positive). This alternative feasibility problem provides us with a concrete understanding of when the robot cannot remain standing; exactly one of (11) and (12) are true. In words, the robot will fall in the current configuration, if there is a local abdomen motion $V_{\mathcal{W} \mathcal{A}}^{\mathcal{A}}$, that generates more power under influence of the gravity wrench than can be provided by the joints. Moreover, this motion is not limited to be one that is produced by the joints, but can also result from slipping at the feet. The slip is encoded by the contact velocity $\nu$.

Although we used these feasibility problems to gain intuition, both the original and alternative problems can be solved computationally to obtain contact forces for planning. Moreover, the problems can be assigned objectives to obtain optimal contact forces. For grasping, examples and procedures for doing this, using Linear Matrix Inequalities, are given in [24], [25]. Similar approaches may be taken for legged robots.

\section{EXAMPLE}

This section will apply some of the concepts from previous sections to illustrate the kinds of tasks which are suitable for the proposed method, and the solutions the produces. In particular, we apply the optimization problem (10) to the redundant planar mobile-manipulator shown in Fig. 5. This manipulator is capable of walking quasistatically, and manipulating while standing. Gravity, $\mathcal{G}$, acts downward. This example is an extension of the one in [1].

Suppose this manipulator is tasked with painting a compliant ceiling. Although most ceilings are very stiff, we can lump the stiffness of the roller and ceiling into one. The task requires pushing upward, and moving side to side. Because the associated twist and wrench are reciprocal, this task will satisfy local compatibility throughout execution.

\footnotetext{
${ }^{6}$ For a more detailed explatation, plase see [23].
} 


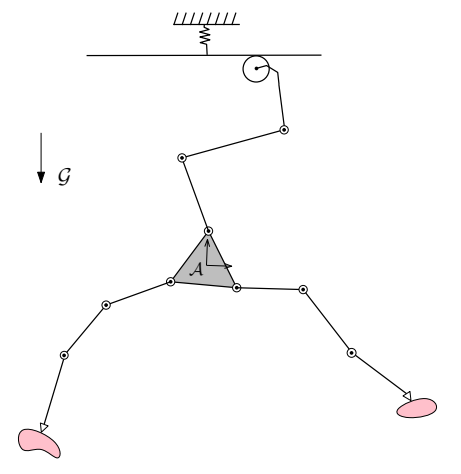

Fig. 5. Redundant Planar Walker

Suppose that the end-effector begins at $x=-1.5$ and must move to $x=1$, and in the process achieve a force of $1 N$ upward. We assume that the ceiling's stiffness is $k_{c}=$ $4 \mathrm{Nm}^{-1}$ (the stiffness is intentionally low for the purposes of illustration). Iteratively applying the solution to (10) with $\alpha=\beta=10$ and $\gamma=0.1$ results in the motion shown in Fig. 6. The resulting motion is smooth and uses all of the body's degrees of freedom to achieve the task, while remaining balanced. The initial mechanism position is shown in blue, while the final one is red.

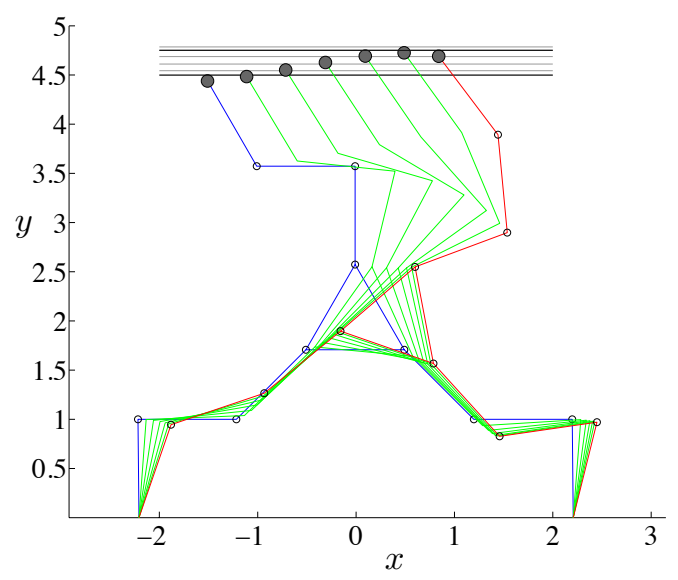

Fig. 6. Simulated Solution

\section{CONCLUSION}

This work studied the kinematics and feasibility of of combined force and position control for multi-limbed robots. We used a classical framework for the combination of forces and positions, and provide a novel compliance model to relate forces to motion. With these assumptions, we first considered the local planning problem for combined force/position tasks with a fixed-base manipulator. We then developed some necessary kinematic extensions for multilimbed robots, and posed the corresponding local planning problem as a constrained optimization.

To gain intuition, we defined and analyzed stance and wrench feasibility, and obtained conditions under which they are possible or infeasible. Finally, we provided a simple example with a planar walker.

\section{REFERENCES}

[1] K. Shankar and J. Burdick, "Kinematics and methods for combined quasi-static stance/reach planning in multi-limbed robots," in IEEE Int. Conf. Robotics and Automation, Hong Kong, 2014.

[2] D. Whitney, "Historical-perspective and state-of-the-art in robot force control," Int. J. Robotics Research, vol. 6, no. 1, pp. 3-14, Spring 1987.

[3] M. Raibert and J. Craig, "Hybrid position/force control of manipulators," ASME J. Dyn. Syst. Measurment Control, vol. 103, pp. 126-133, 1981.

[4] N. Hogan, "Impedance control: An approach to manipulation," ASME J. Dyn. Syst. Measurment Control, vol. 107, pp. 1-7, Mar. 1985.

[5] J. Salisbury and J. Craig, "Articulated hands: Force control and kinematic issues," Int. J. Robotics Research, vol. 1, no. 1, pp. 4-17, Mar. 1982.

[6] J. Salisbury, "Active stiffness control of a manipulator in cartesian coordinates," in IEEE Conf. Decision Control, Dec. 1980, pp. 95-100.

[7] O. Khatib, "A unified approach for motion and force control of robot manipulators," IEEE Trans. Robotics and Automation, vol. 3, no. 1, pp. 43-53, Feb. 1987.

[8] K. Shankar, J. Burdick, and N. Hudson, "A quadratic programming approach to quasi-static whole-body manipulation," in Workshop Algorithmic Foundations Robotics, Istanbul, 2014.

[9] E. Yoshida, M. Poirier, J.-P. Laumond, O. Kanoun, F. Lamiraux, R. Alami, and K. Yokoi, "Whole-body motion planning for pivoting based manipulation by humanoids," in IEEE Int. Conf. Robots and Automation, May 2008, pp. 3181-3186.

[10] Y. Yamamoto and X. Yun, "Coordinating locomotion and manipulation of a mobile manipulator," in IEEE Conf. Decision and Control, 1992, pp. $2643-2648$

[11] O. Khatib, "Mobile manipulation: The robotic assistant," Robotics and Autonomous Systems, vol. 26, no. 2-3, pp. 175-183, 1999.

[12] O. Brock, O. Khatib, and S. Viji, "Task-consistent obstacle avoidance and motion behavior for mobile manipulation," in Proc. IEEE Int. Conf. Robotics and Automation, 2002, pp. 388-393.

[13] K. Inoue, H. Yoshida, T. Arai, and Y. Mae, "Mobile manipulation of humanoids: Real-time control based on manipulabitliy and stability," in IEEE Int. Conf. Robotics and Automation, San Francisco, May 2000, pp. 2217-2222.

[14] M. Gienger, M. Toussaint, and C. Goerick, "Task maps in humanoid robot manipulation," in IEEE/RSJ Int. Conf. Intell. Robots and Systems, Sept. 2008, pp. 2758-2764.

[15] K. Harada, S. Kajita, F. Kanehiro, K. Fujiwara, K. Kaneko, K. Yokoi, and H. Hirukawa, "Real-time planning of humanoid robot's gait for force-controlled manipulation," IEEE/ASME Trans. on Mechatronics, vol. 12, no. 1, pp. 53-62, 2007.

[16] S. Kuindersma, F. Permenter, and R. Tedrake, "An efficiently solvable quadratic program for stabilizing dynamic locomotion," arXiv preprint arXiv:1311.1839, 2013.

[17] B. J. Stephens and C. G. Atkeson, "Dynamic balance force control for compliant humanoid robots," in Intelligent Robots and Systems (IROS), 2010 IEEE/RSJ International Conference on. IEEE, 2010, pp. 1248-1255.

[18] J. Selig and P. McAree, "A simple approach to invariant hybrid control," in IEEE Int. Conf. Robotics and Automation, Minneapolis, MN, 1996.

[19] R. M. Murray, Z. Li, and S. S. Sastry, A mathematical introduction to robotic manipulation. CRC press, 1994.

[20] M. Ohwovoriole and B. Roth, "An extension of screw theory," ASME J. Mechanical Design, vol. 103, no. 4, pp. 725-735, 1981.

[21] J. Selig, "The spatial stiffness matrix from simple stretched springs," in IEEE Int. Conf. Robotics and Automation, San Francisco, 2000.

[22] J. Angeles, "On the nature of the cartesian stiffness matrix," vol. 3, no. 5, pp. 163-170, 2010.

[23] S. P. Boyd and L. Vandenberghe, Convex optimization. Cambridge university press, 2004.

[24] L. Han, J. C. Trinkle, and Z. X. Li, "Grasp analysis as linear matrix inequality problems," Robotics and Automation, IEEE Transactions on, vol. 16, no. 6, pp. 663-674, 2000.

[25] S. P. Boyd and B. Wegbreit, "Fast computation of optimal contact forces," Robotics, IEEE Transactions on, vol. 23, no. 6, pp. 11171132, 2007. 